\title{
High-viscosity glass-ionomer vs. composite resin restorations in persons with disability: Five-year follow-up of clinical trial
}

\section{Gustavo Fabián MOLINA(a) \\ Denise FAULKS ${ }^{(b)}$ \\ Jan MULDER ${ }^{(c)}$ \\ Joannes Elisabert FRENCKEN(d)}

(a) Universidad Nacional de Córdoba, The Dental Faculty, Adress: Haya de la Torre s/n Ciudad Universitaria (5000) Córdoba, Argentina.

(b) Université Clermont Auvergne, CROC EA4847, 63000 Clermont-Ferrand, France: denise.faulks@uca.fr

(c)Radboud University Medical Centre, Radboud Institute for Health Sciences, Department of Dentistry - Oral Function and Prosthetic Dentistry, Nijmegen, The Netherlands: jalma@home.nl

(d)Radboud University Medical Centre, Radboud Institute for Health Sciences, Department of Dentistry - Oral Function and Prosthetic Dentistry, Nijmegen, The Netherlands: jo.frencken@radboudumc.nl

Declaration of Interests: The authors certify that they have no commercial or associative interest that represents a conflict of interest in connection with the manuscript.

Corresponding Author:

Gustavo Fabián Molina

E-mail: gfmolina@hotmail.com

hitps://doi.org/10.1590/1807-3107bor-2019.vol33.0099

Submitted: April 21, 2019

Accepted for publication: September 18, 2019

Last revision: September 27, 2019
Abstract: The aim of this clinical trial was to compare the 5-year cumulative survival of atraumatic restorative treatment restorations using high-viscosity glass-ionomer restorations (ART/HVGIC) and conventional resin composite restorations (CRT) placed in patients with intellectual and/or physical disability. Patients referred for restorative care to a special care service in Córdoba, Argentina, were recruited. Patients and/or caregivers were provided with written and verbal information regarding treatment options and selected the alternative they preferred. The treatment protocols were ART (hand instruments/HVGIC) in the clinic or CRT (rotary instrumentation/resin composite) in the clinic or under general anaesthesia (GA). Two independent, trained and calibrated examiners evaluated restoration survival using established ART codes after 6, 12, 24, 36 and 60 months. The proportional hazard model with frailty corrections provided survival estimates. Jackknife errors were used to test 5-year results. Sixty-six patients (13.6 \pm 7.8 years) with 16 different medical conditions participated. CRT in the clinic proved feasible for five patients (13\%), and 14 patients received CRT under GA (21\%). ART was provided for 47 patients (71.2\%). A total number of 298 dentine carious lesions were restored in primary and permanent teeth (182 ART; 116 CRT). Four patients died between the 3 and 5-year follow up. Percentage survival and jackknife standard error were calculated and were significantly higher for all ART/HVGIC restorations $(90.2 \% \pm 2.6)$ than for all CRT restorations $(82.8 \% \pm 5.3), 5$ years after placement $(p=0.044)$. These 5 -year follow-up results confirm that ART/HVGIC is an effective treatment protocol for patients with disability, equal to that of conventional resin composite restoration. The results of this clinical trial support the use of ART as an evidence-based treatment resource contributing to the reduction of inequalities in access to oral health care among people with disability.

Keywords: Glass Ionomer Cement; Composite Resins; Dental Atraumatic Restorative Treatment; Survival.

\section{Introduction}

Disability is an umbrella term that describes the impairments, activity limitations and participation restrictions experienced by 
a person with a health condition, within their personal and environmental context. ${ }^{1}$ Disabilities may be intellectual, physical, sensory, mental, behavioural, or a mix of these. Receipt of oral health care may be challenging for persons with disability unless the treatment environment is adapted to the individual's specific needs. Common restorative procedures require a patient to be able to cope with the anxiety of the dental setting and the sensation of local anaesthesia, to be able to hold still, to keep the mouth open for prolonged periods, to accept the noise and vibration of the drill and the suction tip, to be able to swallow voluntarily. Patients are required to accept a degree of discomfort and to exert a degree of self-constraint.

Dental materials are designed in vitro and require a controlled environment, particularly the moisture-sensitive composite resins. When controlled operating conditions cannot be attained, routine dental procedures become difficult, compromised or impossible. Optimal clinical operative conditions may be achieved by facilitation techniques, such as sedation or general anaesthesia. Unfortunately, access to such techniques is restricted in many countries or regions, and, where services do exist, they are often reserved for oral surgery. It is thus not surprising that tooth extraction is the most frequently provided dental treatment for patients with disabilities. ${ }^{2}$ Equity in health implies that people in equal need should have equal access, equal treatment and equal treatment outcomes within the health system, regardless of disability status. ${ }^{3,4}$ The question is which dental material is the most appropriate to ensure equal treatment outcomes when operating conditions are difficult?

A review of the literature evidenced the lack of reports on therapeutic strategies for managing carious lesions in people with disability. ${ }^{5}$ The Atraumatic Restorative Treatment (ART) ${ }^{6}$ approach has proven to be an acceptable, feasible and effective technique to prevent/restore cavities in this population. ${ }^{7}$ Manual excavation reduces noise, anxiety and vibration by avoiding the use of rotary instruments and the use of a biological, less moisture-sensitive, high-viscosity glass-ionomer cement (HVGIC) makes ART an interesting alternative to conventional operative treatment. There is evidence that ART reduces the need for restorative care under general anaesthesia. ${ }^{7}$ The survival results reported in meta-analyses on ART restorations in primary and permanent dentitions in different settings are high. ${ }^{8,9}$ These results were reproduced when comparing the survival of ART restorations with conventional resin composite restorations in people with disability at one year ${ }^{10}$ and at 3 years. ${ }^{11}$ Survival percentages of all ART/HVGIC restorations in primary and permanent teeth were significantly higher than comparable resin composite restorations (CRT).

The present study compares the longevity of ART/HVGIC restorations and conventional resin composite restorations (CRT) 5 years after placement in patients with intellectual and/or physical disability, in order to determine the effectiveness of these two restorative treatment options in the long term. The null-hypothesis tested is that there is no significant difference in the survival of all ART/HVGIC restorations in comparison to all CRT/resin composite restorations after 5 years.

\section{Methodology}

The methodology is presented in detail elsewhere, ${ }^{10}$ so only a concise outline is given below.

\section{Ethics}

Ethical approval was obtained from the local Ethical Committee, CIEIS Facultad de Odontología, Universidad Nacional de Córdoba with the reference number 38/2012 and the trial was registered at the Netherlands Trial Register, number 4400.

\section{Participants}

People with intellectual disability (with or without motor impairment) who regularly attend 5 different care institutions in Cordoba, Argentina and who were referred for restorative care to a Special Care Dentistry service held at the Haemophilia Foundation in Córdoba, Argentina, over a six-month period (August 2012 to February 2013) were considered potential participants for inclusion in the study. 


\section{Inclusion criteria}

Patients with a recognised disability, and with at least one dentine carious lesion in a primary or permanent tooth without pulpal compromise and without spontaneous pain or tooth mobility, but in occlusion with the antagonist tooth or teeth and in contact with the neighbouring tooth or teeth, were included in the study. Signed consent was given by the patient or their legal representative.

Patients were examined by one Special Care dentist. A full description of the functional, social and environmental context of the patient was recorded using the International Classification of Functioning (ICF Checklist for Oral Health).,12 Clinical examination included: a) report of pain by the patient and/or caregiver, and targeted examination of potentially painful teeth; b) presence of dental plaque, assessed according to the criteria of Greene and Vermillion and reported using the Simplified Oral Hygiene Index (S-OHI); ${ }^{13} \mathrm{c}$ ) gingival bleeding, measured on buccal and lingual surfaces of all teeth according to the criteria of Ainamo and Bay and reported using the gingival bleeding index $(\mathrm{GBI}) r^{14}$ and d) dental caries with a dmft or DMFT score according to the criteria of the World Health Organization (WHO) ${ }^{15}$

\section{Attribution to treatment groups}

Two different treatment protocols were proposed: a) Atraumatic Restorative Treatment (ART), involving the removal of decayed carious tissue with hand instruments only and restoration of the cavity with an encapsulated high-viscosity glass-ionomer cement, either Chemfil Rock (Dentsply, Konstanz, Germany) or Equia system (GC America, Chicago, USA); and b) Conventional Restorative Treatment (CRT), using rotary instruments for carious tissue removal and restoration of the cavity with an adhesive system and resin composite under rubber dam (Single bond and Filtek Z-350, 3M Espe, St. Paul, USA).

Standardised verbal information and two validated informative brochures were presented to the patient/parent/carer (termed 'participants' from this point) with the treatment options. The dentists were instructed to be as neutral as possible during this presentation. Participants kept the brochures to read at home and at the second visit, requested their choice of either ART treatment or conventional treatment (CRT). Dentists recorded the reasons that led participants to choose either option in order to identify their expectations and perceived barriers to carrying out a dental procedure. The participants' choice led to attribution to one of two treatment groups : an ART group and a CRT group. ${ }^{10}$

\section{Treatment provision}

At the second visit, the operator performed the treatment selected by the participant, resulting in the following situations: a) The patient was able to cope with the dental treatment and the operator was able to place the restorations in optimal conditions. If further restorations were needed, additional sessions were scheduled using the same treatment; b) The patient was unable to cope with the dental treatment and the operator was, therefore, unable to place the restorations in optimal conditions. If further restorations were needed, treatment was programmed using the alternative treatment; c) The patient was unable to cope with either treatment protocol and the patient was referred for conventional treatment under general anaesthesia (GA).

\section{Evaluation}

Suitability of the approaches was analysed in terms of acceptance, feasibility and level of satisfaction after one year of treatment, the results of which are published elsewhere. $^{7}$

Restorations were evaluated by two calibrated independent examiners at 6, 12, 24, 36 and 60 months using established ART restoration assessment criteria. ${ }^{6}$ The inter-examiner consistency, expressed as kappa coefficient and the percentage of agreement $(\mathrm{Po})$ at the 60 months evaluation was 0.84 (95\%CI: 0.77-0.92) and $95 \%$ respectively. One-year and three-year survival data have been previously published. ${ }^{10,11}$

\section{Statistical analyses}

The analyses in the current manuscript present the comparison between the two treatment groups, 
ART/HVGIC and CRT/resin composite restorations, for the variables 'all restorations', 'all single- and all multiple restorations' and 'restorations in permanent teeth'.

Restorations with no defects, or defects of less than $0.5 \mathrm{~mm}$ at the restoration margin, were considered to have survived. All other assessment codes were considered failures. Data were entered into a database and analysed using SAS 9.2 software. The Proportional Hazard Rate Regression Model ${ }^{16}$ with frailty correction ${ }^{17}$ was used to estimate cumulative survival percentages of ART and CRT restorations. The Jackknife method ${ }^{18}$ was applied to calculate standard errors for comparison of the 5-year results. Statistical significance was set at $\alpha=0.05$.

\section{Results}

\section{Disposition of subjects}

A flow diagram is presented in Figure. The sample consisted of 298 restored cavities placed in 66 patients with disability (36 males and 30 females with a mean age of $13.6, \mathrm{SD} \pm 7.8$ years). In the permanent dentition, 122 cavities were restored using ART and 47 using CRT. In the primary dentition 60 cavities were restored using ART and 69 using CRT. Four patients died in the last two years of the 5 year follow up, three from the ART group and one from the CRT group.

\section{Survival of restorations}

\section{All restorations}

Table 1 shows the cumulative survival percentages and Jackknife standard errors of all restorations by treatment group and evaluation interval. There was a statistically significant difference in the 5 -year cumulative survival percentage between all ART/HVGIC restorations $(90.2 \% \pm 2.6)$ and all CRT/resin composite restorations $(82.8 \% \pm 5.3)$ $(\mathrm{p}=0.044)$, with ART restorations surviving longer.

Single- versus multiple-surface restorations

Fifteen ART/HVGIC restorations failed overall, 6 in single-surface and 9 in multiple-surface restorations, whilst in the CRT/resin composite group 4 single-surface and 14 multiple-surface restorations failed over the 5 study years. The cumulative survival percentages and Jackknife standard errors of all restorations by evaluation interval, treatment group and type of surface are presented in Table 2. At 5 years, there was no statistically significant difference between all single-surface ART/HVGIC $(94.2 \% \pm 2.3)$ and all single-surface CRT/resin composite restorations $(93.8 \% \pm 3.0)(\mathrm{p}=0.889)$ and between all multiple-surface ART/HVGIC $(76.4 \% \pm 7.0)$ and all multiple-surface CRT/resin composite restorations $(61.8 \% \pm 8.8)(p=0.107)$.

\section{Restorations in posterior permanent teeth}

The 5 years survival percentage of single-surface CRT/resin composite restorations in posterior permanent teeth (100.0\%) (as opposed to all types of teeth) was statistically significantly higher $(p=0.023)$ than that for comparable ART/HVGIC restorations $(94.6 \%$ : SE $=2.3)$. Although the 5 years survival percentage of multiple-surface ART/HVGIC in posterior permanent teeth was higher $(85.5 \%$ : SE $=7.2)$, the difference with the survival percentage of CRT/resin composite multiple-surface restorations $(66.9 \%: \mathrm{SE}=19.0)$ was not statistically significant $(p=0.12)$.

\section{Materials and conditions of restoration} placement Type of high-viscosity glass-ionomer cement used

There was no statistically significant difference in the 5-year survival percentage of all ART/Equia $(90.6 \% \pm 4.1)$ and ART/Chemfil Rock $(90.1 \% \pm 3.8)$ for permanent restorations $(p=0.896)($ Table 3$)$.

\section{Placement of composite under general anaesthesia or in the office}

A statistically significant difference was observed between all CRT/resin composite permanent restorations placed under general anaesthesia $(96.7 \% \pm 3.3)$ and those placed in a conventional dental setting $(64.7 \% \pm 11.6)(\mathrm{p}<0.001)$ (Table 4$)$, with the restorations placed under general anaesthesia surviving longer. 


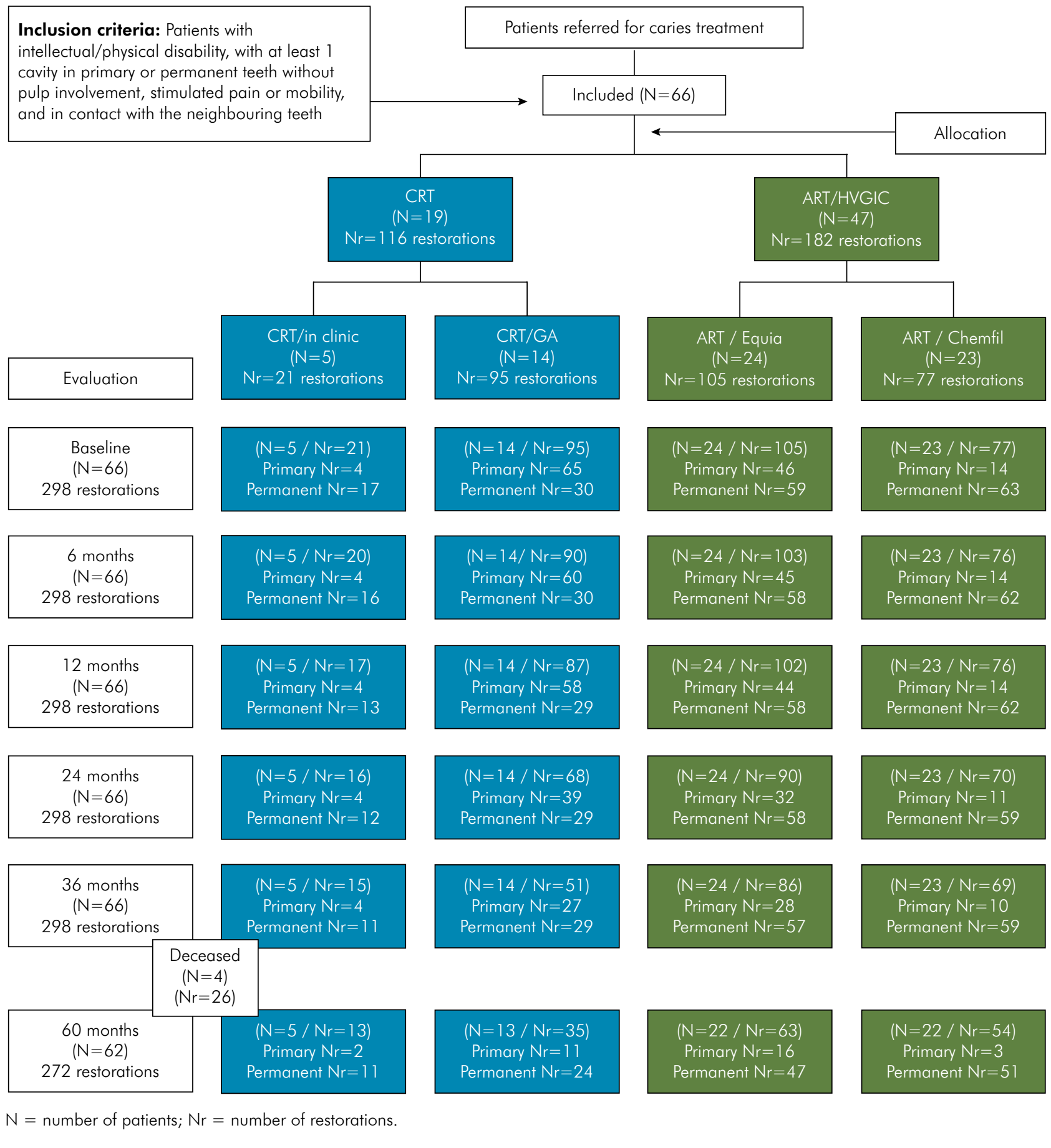

Figure. Flow-chart of the 5-year follow-up of patients with disability that received ART and CRT restorations.

\section{Discussion}

\section{Methodology}

The main challenge in the design of this clinical trial was to ensure randomisation without encroaching on the right to choice of treatment, particularly in a vulnerable population unable for the most part to make personal treatment decisions. This difficulty was overcome by allowing participants to choose their treatment group. Although this 
High-viscosity glass-ionomer vs. composite resin restorations in persons with disability: Five-year follow-up of clinical trial

Table 1. Cumulative survival percentages, Jackknife standard error (SE) and p-values after 5 years for all restorations placed in patients with disability by treatment group.

\begin{tabular}{|c|c|c|c|c|c|c|c|}
\hline \multirow{3}{*}{$\begin{array}{l}\text { Interval } \\
\text { Months }\end{array}$} & \multicolumn{6}{|c|}{ Treatment group } & \multirow{3}{*}{$p$-value } \\
\hline & \multicolumn{3}{|c|}{ ART/HVGIC $(n=182)$} & \multicolumn{3}{|c|}{ CRT/resin composite $(n=116)$} & \\
\hline & Nfail & Surv (\%) & SE & Nfail & Surv (\%) & SE & \\
\hline 6 & 3 & 98.4 & 1.0 & 6 & 94.8 & 2.4 & 0.024 \\
\hline 12 & 4 & 97.8 & 1.0 & 12 & 89.7 & 3.3 & $<0.001$ \\
\hline 24 & 9 & 94.8 & 2.1 & 15 & 86.6 & 4.1 & 0.005 \\
\hline 36 & 9 & 94.8 & 2.1 & 18 & 82.8 & 5.3 & $<0.001$ \\
\hline 60 & 15 & 90.2 & 2.6 & 18 & 82.8 & 5.3 & 0.044 \\
\hline
\end{tabular}

$\mathrm{n}=$ number of restorations; Nfail $=$ number of failed restorations.

Table 2. Cumulative survival percentages and Jackknife standard error (SE) of all ART/HVGIC and CRT/resin composite restorations by type of surface.

\begin{tabular}{lcccccccc}
\hline \multirow{2}{*}{ Interval } & \multicolumn{3}{c}{ ART/HVGIC $(\mathrm{n}=182)$} & \multicolumn{4}{c}{ CRT/ resin composite $(\mathrm{n}=116)$} \\
\cline { 2 - 9 } Months & \multicolumn{2}{c}{ Single $(\mathrm{n}=142)$} & \multicolumn{2}{c}{ Multiple $(\mathrm{n}=40)$} & \multicolumn{2}{c}{ Single $(\mathrm{n}=71)$} & \multicolumn{2}{c}{ Multiple $(\mathrm{n}=45)$} \\
\hline 6 & Mean & SE & Mean & SE & Mean & SE & Mean & SE \\
12 & 100.0 & 0.0 & 92.5 & 4.2 & 97.2 & 2.0 & 91.1 & 4.2 \\
24 & 100.0 & 0.0 & 90.0 & 4.7 & 97.2 & 2.0 & 77.8 & 6.2 \\
36 & 99.2 & 0.8 & 79.7 & 6.4 & 93.8 & 3.0 & 75.1 & 6.5 \\
60 & 99.2 & 0.8 & 79.7 & 6.4 & 93.8 & 3.0 & 61.8 & 8.8 \\
\hline
\end{tabular}

$\mathrm{n}=$ number of restorations; ${ }^{a, b, c, d} p$-values: $p^{a, b}=0.889 ; p^{c, d}=0.107$

Table 3. Cumulative survival percentages and Jackknife standard error (SE) of ART restorations in permanent teeth of patients with disability after 5 years by brand of high-viscosity glass-ionomer cement (HVGIC).

\begin{tabular}{|c|c|c|c|c|c|c|c|}
\hline \multirow{3}{*}{$\begin{array}{l}\text { Interval } \\
\text { Months }\end{array}$} & \multicolumn{6}{|c|}{ ART/HVGIC } & \multirow{3}{*}{$p$-value } \\
\hline & \multicolumn{3}{|c|}{ Equia $(n=59)$} & \multicolumn{3}{|c|}{ Chemfil rock $(n=63)$} & \\
\hline & Nfail & Surv (\%) & SE & Nfail & Surv (\%) & SE & \\
\hline 6 & 1 & 98.3 & 1.7 & 1 & 98.4 & 1.6 & 0.789 \\
\hline 12 & 1 & 98.3 & 1.7 & 1 & 98.4 & 1.6 & 0.789 \\
\hline 24 & 1 & 98.3 & 1.7 & 4 & 93.6 & 3.1 & 0.124 \\
\hline 36 & 1 & 98.3 & 1.7 & 4 & 93.6 & 3.1 & 0.124 \\
\hline 60 & 5 & 90.6 & 4.1 & 6 & 90.1 & 3.8 & 0.896 \\
\hline
\end{tabular}

$\mathrm{n}=$ number of restorations; Nfail $=$ number of failed restorations.

Table 4. Cumulative survival percentages and Jackknife standard error (SE) of CRT/resin composite restorations placed in permanent teeth of patients with disability after 5 years by setting.

\begin{tabular}{|c|c|c|c|c|c|c|c|}
\hline \multirow{3}{*}{$\begin{array}{l}\text { Interval } \\
\text { Months }\end{array}$} & \multicolumn{6}{|c|}{ CRT/resin composite - setting } & \multirow{3}{*}{$p$-value } \\
\hline & \multicolumn{3}{|c|}{ CRT-in office $(n=17)$} & \multicolumn{3}{|c|}{ CRT-general anaesthesia $(n=30)$} & \\
\hline & Nfail & Surv (\%) & SE & Nfail & Surv (\%) & SE & \\
\hline 6 & 1 & 94.1 & 5.7 & 0 & 100.0 & 0.0 & $<0.001$ \\
\hline 12 & 4 & 76.5 & 10.2 & 1 & 96.7 & 3.3 & 0.009 \\
\hline 24 & 5 & 70.6 & 11.0 & 1 & 96.7 & 3.3 & 0.001 \\
\hline 36 & 6 & 64.7 & 11.6 & 1 & 96.7 & 3.3 & $<0.001$ \\
\hline 60 & 6 & 64.7 & 11.6 & 1 & 96.7 & 3.3 & $<0.001$ \\
\hline
\end{tabular}

$\mathrm{n}=$ number of restorations; Nfail $=$ number of failed restorations. 
weakened the power of the results related to the longevity of the restorations, the study data still allow a useful comparison between treatment techniques and materials.

The trial sustained no drop-outs up to the 3-year evaluation. Between the 3 and 5-year evaluation, 4 participants died. The Proportional Hazard Model compensated for this effect on analysis and the Jackknife method adjusted for the effect of multiple restorations per participant. The internal validity of the study may be considered high due to the low number of drop-outs, and the fact that the provision of care for people with disability in the present trial is in line with that available generally in Argentina. The external validity of the 5-year trial is lower, in that different health systems in other world centres may have different approaches to dental treatment for persons with disability, but the outcomes are still of relevance for policy makers and clinicians in countries outside of Argentina.

\section{Main findings}

The null-hypothesis was rejected. A significant difference in the survival percentages of all ART/HVGIC and all CRT/resin composite restorations after 5 years was found, with ART/HVGIC restorations surviving longer. This is an important outcome as the trial was designed to investigate a new treatment concept in caries care (ART) for use in people with a disability. The fact that participants chose ART as their preferred treatment method, ${ }^{7}$ added to the higher 5-year survival of ART restorations compared to conventional resin restorations, indicates that Special Care dentists should consider choosing ART more frequently as the most appropriate treatment approach for their patients. This will require a change in clinical practice and a shift in attitudes towards ART, which is still considered by some to be a 'lower quality' treatment option, appropriate only for developing countries. ${ }^{19}$ Restorative techniques and materials need to be adapted to individual patient context, whilst maintaining equal treatment outcomes. This study has demonstrated one way in which this may be achieved.

Paradigm shifts in dentistry are ongoing in all domains. Restorative dentistry has navigated from mineral-based, to metal-based, and then to resin-based restorative materials, slightly returning to mineral-based materials in order to provide a replacement for lost structures that comply with biological, mechanical and aesthetic requirements. The high longevity of amalgam restorations is currently being reached by tooth-coloured products ${ }^{20}$ providing improved aesthetics, but the newer materials are still struggling to sufficiently resist biting forces and are sensitive to operating conditions. ${ }^{21}$ If only restorations in posterior permanent teeth are considered, resin composite restorations survived significantly longer in single-surface cavities (100\%) than comparable ART/HVGIC restorations ( $94.6 \% \mathrm{SE}=2.3$ ) after 5 years $(p=0.023)$. Although not significantly different, the 5-year survival percentage of multiple-surface ART/HVGIC restorations in posterior permanent teeth $(85.5 \% \mathrm{SE}=7.2)$ was higher than for comparable resin composite restorations $(66.9 \% \mathrm{SE}=19.0)(\mathrm{p}=0.12)$. These findings for multiple-surface restorations reflect the improved performance of the more recent capsulated HVGIC materials.

Attitudes to the provision of restorative dentistry in adults with disability have also evolved. Anecdotal information and older publications ${ }^{22}$ assert that amalgam is the most appropriate restorative material to restore posterior permanent teeth in people with physical or intellectual disability. Such practice still prevails, as a retrospective audit of restorative procedures carried out under general anaesthesia in special needs patients reveals. ${ }^{23}$ In this study, amalgam was the material of choice for patients above 12 years of age. The use of amalgam will however soon become impossible in many countries and Special Care dentists need to be prepared to change their therapeutic arsenal.

Main concerns for the longevity of direct restorations in patients with disability are related to the mechanical properties of the restorative material. Motor impairment leads to a high prevalence of para-functional activity in this population. ${ }^{24}$ In addition, functional tooth surfaces are essential for maintaining masticatory capacity and mandibular stability on swallowing, both of which may be altered in the presence of neuromotor impairment. ${ }^{25}$ It is not sufficient to 'cure the cavity' by placing a 
non-functional restoration that cannot withstand occlusal force or wear, even if the restoration has good long-term survival in terms of retention and prevention of recurrent caries activity. For the present study, two brands of high-viscosity glass-ionomer cement were selected following preliminary in-vitro tests to determine the best mechanical performance. ${ }^{26,27}$ Outcomes from different clinical trials demonstrate acceptable properties of high-viscosity glass-ionomer cements compared to resin composite in single- and multiple-surface restorations in posterior permanent teeth. ${ }^{28,29}$ These aspects require further research attention, however, in the population with disability.

Low survival percentages in multiple-surface restorations for both CRT and ART/HVGIC groups in the current study lead to reflection regarding appropriate treatment strategies for large restorations. Two different studies have shown that stainless steel crowns placed in primary teeth of non-disabled patients under general anaesthesia were the most reliable restorations, while composite restorations were the least durable. ${ }^{30,31}$ Stainless steel crowns are increasingly being used in the permanent dentition in this context, and may replace amalgam as an alternative to large multi-surface direct restoration, particularly after pulp treatment. ${ }^{32,33}$ This strategy needs to be evaluated further.

The effectiveness of restorations provided under sedation and under general anaesthesia has been investigated. A recent clinical study that compared high-viscosity glass-ionomer to composite restorations placed in primary teeth of patients with disability under general anaesthesia, reported low failure rates for both materials in the two groups. ${ }^{33}$ These results differ from the findings of the present study, which show higher survival percentages for all HVGICs (placed in the office), compared to all resin composites placed either in the office or under general anaesthesia, in primary and permanent teeth.

In addition, significantly lower survival percentages were found for resin composite restorations placed in permanent teeth in the dental office compared to placement under general anaesthesia. It is likely that this reflects the ability for better moisture control under general anaesthesia, despite the fact that rubber dam was used systematically in this trial, both in the operating room and in the office.

\section{Conclusion}

High-viscosity glass-ionomer cement restorations placed using the ART method show higher longevity than conventional composite resin restorations overall in persons with disability. Significantly lower survival percentages were reported for multiple surface than single surface restorations, for both ART/HVGIC and CRT. In order to respect the right to equal treatment outcomes in this population, Special Care dentists need to take these findings into account when treatment planning for persons with disability.

\section{References}

1. World Health Organisation. International Classification of Functioning, Disability and Health (ICF). Geneva, Switzerland: WHO, 2001.

2. McKelvey VA, Morgaine KC, Thomson WM. Adults with intellectual disability: a mixed-methods investigation of their experiences of dental treatment under general anaesthetic. N Z Dent J. 2014 Jun;110(2):58-64.

3. Boeckxstaens P, Smedt DD, Maeseneer JD, Annemans L, Willems S. The equity dimension in evaluations of the quality and outcomes framework: a systematic review. BMC Health Serv Res. 2011 Aug;11(1):209. https://doi.org/10.1186/1472-6963-11-209

4. Faulks D, Fabián Molina G. How to provide the evidence base for techniques and interventions that ensure equal treatment outcomes for people with disability? Spec Care Dentist. 2018 May;38(3):119-20. https://doi.org/10.1111/scd.12285

5. Molina GF, Leal S, Frencken JE. Strategies for managing carious lesions in patients with disabilities: a systematic review. J Disabil Oral Health. 2011;12:159-67.

6. Frencken JE, Holmgren CJ. Atraumatic Restorative Treatment (ART) for dental caries.d. Nijmegen: STI Book; 1999.

7. Molina GF, Faulks D, Frencken J. Acceptability, feasibility and perceived satisfaction of the use of the Atraumatic Restorative Treatment approach for people with disability. Braz Oral Res. 2015;29(1):1-9. https://doi.org/10.1590/1807-3107BOR-2015.vol29.0097 
8. Amorim RG, Leal SC, Frencken JE. Survival of atraumatic restorative treatment (ART) sealants and restorations: a meta-analysis. Clin Oral Investig. 2012 Apr;16(2):429-41. https://doi.org/10.1007/s00784-011-0513-3

9. de Amorim RG, Frencken JE, Raggio DP, Chen X, Hu X, Leal SC. Survival percentages of atraumatic restorative treatment (ART) restorations and sealants in posterior teeth: an updated systematic review and meta-analysis. Clin Oral Investig. 2018 Nov;22(8):2703-25. https://doi.org/10.1007/s00784-018-2625-5

10. Molina GF, Faulks D, Mazzola I, Mulder J, Frencken JE. One year survival of ART and conventional restorations in patients with disability. BMC Oral Health. 2014 May;14(1):49. https://doi.org/10.1186/1472-6831-14-49

11. Molina GF, Faulks D, Mazzola I, Cabral RJ, Mulder J, Frencken JE. Three-year survival of ART high-viscosity glass-ionomer and resin composite restorations in people with disability. Clin Oral Investig. 2018 Jan;22(1):461-7. https://doi.org/10.1007/s00784-017-2134-y

12. Faulks D, Norderyd J, Molina G, Macgiolla Phadraig C, Scagnet G, Eschevins C, et al. Using the International Classification of Functioning, Disability and Health (ICF) to describe children referred to special care or paediatric dental services. PLoS One. 2013 Apr;8(4):e61993. https://doi.org/10.1371/journal.pone.0061993

13. Greene JC, Vermillion JR. The simplified oral hygiene index. JAm DentAssoc. 1964 Jan;68(1):7-13. https://doi.org/10.14219/jada.archive.1964.0034

14. Ainamo J, Bay I. Problems and proposals for recording gingivitis and plaque. Int Dent J. 1975 Dec;25(4):229-35.

15. World Health Organisation. Oral health surveys, basic methods 4. Geneva: World Health Organization; 1997.

16. CoxDR. Regression models and life tables (with discussion). JRStat Soc B. 1972;34(2):187-220. https://doi.org/10.1111/j.2517-6161.1972.tb00899.x.

17. Efron B. The jackknife, the bootstrap, and other resampling plans. Philadelphia: SIAM-NSF; 1982. https://doi.org/10.1137/1.9781611970319

18. Hougaard P. Frailty models for survival data. Lifetime Data Anal. 1995;1(3):255-73.

19. Molina GF, Faulks D, Frencken JE. Suitability of ART approach for managing caries lesions in people with disability-Experts' opinion. Acta Odontol Scand. 2013;71(6):1430-5. https://doi.org/10.3109/00016357.2013.766361

20. Ástvaldsdóttir Á, Dagerhamn J, van Dijken JW, Naimi-Akbar A, Sandborgh-Englund G, Tranæus S, et al. Longevity of posterior resin composite restorations in adults - A systematic review. J Dent. 2015 Aug;43(8):934-54. https://doi.org/10.1016/i.ident.2015.05.001

21. Demarco FF, Corrêa MB, Cenci MS, Moraes RR, Opdam NJ. Longevity of posterior composite restorations: not only a matter of materials. Dent Mater. 2012 Jan;28(1):87-101. https://doi.org/10.1016/i.dental.2011.09.003

22. Schulz P. [The possibility of oral rehabilitation of the handicapped under anesthesia]. Dtsch Zahnarztl Z. 1977 Feb;32(2):127-9. German.

23. Mallineni SK, Yiu CK. A Retrospective Audit of Dental Treatment Provided to Special Needs Patients under General Anesthesia During a Ten-Year Period. J Clin Pediatr Dent. 2018;42(2):155-60. https://doi.org/10.17796/1053-4628-42.2.13

24. Andersson-Norinder J, Sjogreen L. Orofacial dysfunction. In: Nunn J, editor. Disability and oral care. London: FDI World Dental Press; 2000. p. 104-14.

25. Hennequin M, Mazille MN, Cousson PY, Nicolas E. Increasing the number of inter-arch contacts improves mastication in adults with Down syndrome: a prospective controlled trial. Physiol Behav. 2015 Jun;145:14-21. https://doi.org/10.1016/i.physbeh.2015.03.034

26. Molina GF, Cabral RJ, Mazzola I, Lascano LB, Frencken JE. Mechanical performance of encapsulated restorative glass-ionomer cements for use with Atraumatic Restorative Treatment (ART). J Appl Oral Sci. 2013;21(3):243-9. https://doi.org/10.1590/1679-775720130129

27. Fabián Molina G, Cabral RJ, Mazzola I, Brain Lascano L, Frencken JE. Biaxial flexural strength of high-viscosity glass-ionomer cements heat-cured with an LED lamp during setting. BioMed Res Int. 2013;2013:838460. https://doi.org/10.1155/2013/838460

28. Gurgan S, Kutuk ZB, Ergin E, Oztas SS, Cakir FY. Four-year randomized clinical trial to evaluate the clinical performance of a glass ionomer restorative system. Oper Dent. 2015 Mar-Apr;40(2):134-43. https://doi.org/10.2341/13-239-C

29. Menezes-Silva R, Velasco SR, Bastos RS, Molina G, Honório HM, Frencken JE, et al. Randomized clinical trial of class II restoration in permanent teeth comparing ART with composite resin after 12 months. Clin Oral Investig. 2019 Sep;23(9):3623-35. https://doi.org/10.1007/s00784-018-2787-1

30. Tate AR, Ng MW, Needleman HL, Acs G. Failure rates of restorative procedures following dental rehabilitation under general anesthesia. Pediatr Dent. 2002 Jan-Feb;24(1):69-71.

31. Al-Eheideb AA, Herman NG. Outcomes of dental procedures performed on children under general anesthesia. J Clin Pediatr Dent. 2003;27(2):181-3. https://doi.org/10.17796/icpd.27.2.k3307186n7086r11

32. Cousson PY, Nicolas E, Hennequin M. A follow-up study of pulpotomies and root canal treatments performed under general anaesthesia. Clin Oral Investig. 2014 May;18(4):1155-63. https://doi.org/10.1007/s00784-013-1090-4

33. Pani SC. Comparison of high viscosity glass ionomer cement to composite restorations placed in primary teeth under general anesthesia. Pediatr Dental J. 2018;28(3):154-9. https://doi.org/10.1016/i.pdj.2018.08.001 